\title{
The Role of Polypodium leucotomos Extract in the Treatment of Porphyria Cutanea Tarda
}

\author{
Michael Hatch ${ }^{1}$, Lisa Mask-Bull2*, Michelle B. Tarbox ${ }^{3}$ \\ ${ }^{1}$ Department of Internal Medicine, Internal Medicine at the University of Alabama Medical Center, Mobile, Alabama \\ ${ }^{2}$ Department of Dermatology, Dermatology at Texas Tech Health Sciences Center, Lubbock, USA \\ ${ }^{3}$ Department of Dermatology, Clinical Dermatology and Dermatopathology at Texas Tech University Health Sciences Center, \\ Lubbock, USA \\ Email: ^Lisa.Mask@ttuhsc.edu
}

How to cite this paper: Hatch, M., MaskBull, L. and Tarbox, M.B. (2017) The Role of Polypodium leucotomos Extract in the Treatment of Porphyria Cutanea Tarda. Open Access Library Journal, 4: e2858.

https://doi.org/10.4236/oalib.1102858

Received: June 28, 2016

Accepted: July 25, 2017

Published: July 28, 2017

Copyright $\odot 2017$ by authors and Open Access Library Inc.

This work is licensed under the Creative Commons Attribution International License (CC BY 4.0).

http://creativecommons.org/licenses/by/4.0/ (c) (i) Open Access

\begin{abstract}
The dermatologic manifestations of porphyrias result from localized cutaneous formation of reactive oxygen species. The significance of these inflammatory mediators may extend beyond the skin, as increased oxidative stress may play a role in the systemic manifestations of porphyrias. Polypodium leucotomos is a tropical fern that has demonstrated antioxidant, anti-inflammatory and photoprotective properties. Although it has proven effective in the treatment of various photoaggravated dermatoses, we are unaware of any prior reports citing Polypodium leucotomos as a beneficial treatment option in the setting of porphyria cutanea tarda. We describe a patient with refractory porphyria cutanea tarda, who demonstrated dramatic improvement in cutaneous lesions following the initiation of Polypodium leucotomos extract. Due to its low toxicity and demonstrated direct interference in the production of inflammatory mediators are central to cutaneous manifestations of porphyrias, we propose consideration of Polypodium leucotomos as a potential therapeutic option for patients with porphyria cutanea tarda.
\end{abstract}

\section{Subject Areas}

Dermatology

\section{Keywords}

Heliocare, Polypodium leucotomos Extract, Porphyria

\section{Introduction}

Multiple culprits are central to the pathogenesis of the porphyrias including reactive oxygen species, matrix metalloproteinases, mast cells, polymorphonuc- 
lear cells, fibroblasts, erythrocytes and members of the complement pathway [1]. Reactive oxygen species specifically are thought to cause the majority of cellular and tissue damage seen in these conditions [1]. The porphyrias result from dysfunction of enzymes involved in the production of heme which leads to the accumulation of upstream porphyrins [1]. When exposed to wavelengths between $400-410 \mathrm{~nm}$, porphyrins intensely absorb light energy and reach an excited state from which energy is subsequently transferred to oxygen molecules, creating reactive oxygen species [1]. The singlet oxygen species and free radicals produced by photoactivated porphyrins lead to lipid peroxidation and protein cross-linking, resulting in significant tissue damage [1]. The dermatologic manifestations of the porphyrias are dependent on the solubility of the accumulated porphyrins. Cutaneous porphyrias, such as porphyria cutanea tarda (PCT), are characterized by the accumulation of water-soluble porphyrins in the skin leading to the formation of vesicles and bullae on sun-exposed skin.

Polypodium leucotomos ( $P$. leucotomos) is a tropical fern that has demonstrated antioxidant, anti-inflammatory and photoprotective properties [2]. $P$. leucotomos inhibits ultraviolet radiation (UVR) induced production of reactive oxygen species and lipid peroxidation [2]. Additionally $P$. leucotomos has been shown to improve membrane integrity, stimulate elastin expression and inhibit matrix metalloproteinase-1 expression in UVR exposed fibroblasts and keratinocytes [3]. Clinically, the photoprotective effects of $P$. leucotomos have treatment benefits for several cutaneous diseases aggravated by UVR exposure, including polymorphous light eruption and other idiopathic photodermatoses. We describe a patient with PCT who failed to respond to traditional therapy but demonstrated dramatic improvement in cutaneous lesions following the initiation of P. leucotomos extract.

\section{Case Presentation}

A 61-year-old male with history of end stage renal disease (ESRD) requiring hemodialysis (HD) and hepatitis C presented with a two week history of painful vesicles, bullae and erosions located on the bilateral dorsal hands and forearms which were exacerbated with sun exposure (Figure 1). Dermatologic examina-

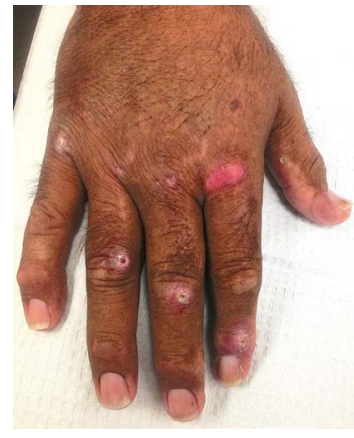

(a)

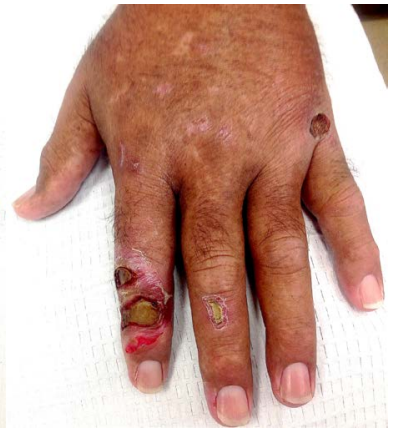

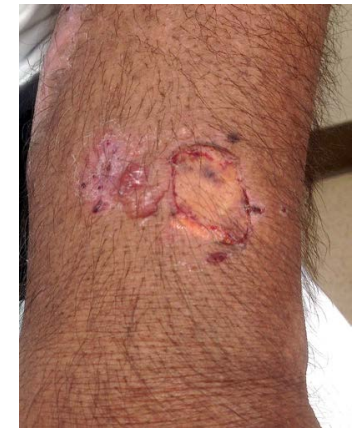

(b)

Figure 1. Clinical appearance of porphyria cutanea tarda lesions before treatment. Erosions, ulcers and crusted papules of the bilateral dorsal hands and extensor forearms are evident. 
tion revealed erosions, crusted papules and tense bullae of the bilateral extensor arms and hands. Histopathologic examination of a $4 \mathrm{~mm}$ punch biopsy of the left forearm demonstrated a pauciinflammatory subepidermal bullae and festooning of the dermal papillae. Direct immunofluorescence demonstrated perivascular deposition of $\operatorname{IgM}, \operatorname{IgG}$ and C3 in the superficial dermis. A 24 hour urine porphyrin profile was notable for elevated total porphyrins with excess excretion of urinary uroporphyrin, coproporphyrin and heptacarboxyl porphyrin. The suspected diagnosis of PCT was confirmed by the detection of fecal isocoproporphyrin. Therapy with hydroxychloroquine $400 \mathrm{mg}$ once weekly after hemodialysis was initiated. The patient adhered to this regimen for nine months and exercised strict sun avoidance. Despite compliance with these measures, the patient demonstrated no improvement and continued to develop multiple painful bullae on the extensor arms. Follow up examination of the bilateral upper extremities confirmed the presence of erosions, sclerotic plaques and over twenty vesicles $1-3 \mathrm{~mm}$ in size. Topical triamcinolone $0.1 \%$ ointment BID and mupirocin $2 \%$ ointment daily were added to his existing regimen of hydroxychloroquine and a three month follow up examination was recommended. At this subsequent examination the patient again demonstrated no improvement despite compliance with all recommendations, including strict sun avoidance and hydroxychloroquine for a period of 12 months. P. leucotomos extract (Heliocare) $240 \mathrm{mg}$ PO once in the AM was then added to the pre-existing regimen. Three months following initiation of Heliocare, the patient endorsed a $90 \%$ reduction in cutaneous lesions and near complete resolution of pain. Physical examination demonstrated just two vesicles on the right dorsal hand (Figure 2). The patient has been taking $P$. leucotomos extract for a period of 15 months and continues to experience sustained improvement without exacerbation.

\section{Discussion}

It is our opinion that the addition of $P$. leucotomos was at least partially responsible for the dramatic dermatologic improvement demonstrated by our patient. The patient consistently exercised sun avoidance, his liver function remained

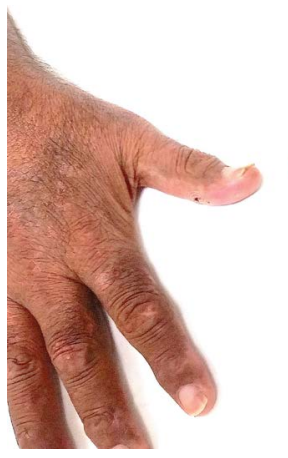

(a)

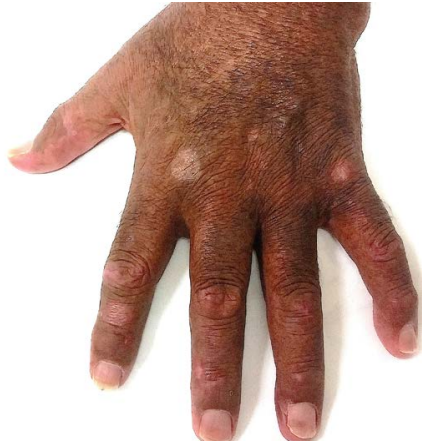

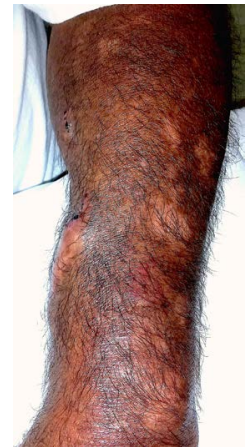

(b)

Figure 2. Improvement in porphyria cutanea tarda lesions following treatment with $P o$ lypodium leucotomos extract. Healing erosions and scars are noted on the bilateral dorsal hands and extensor forearm. 
stable and he was compliant with hydroxychloroquine for 12 months, yet he continued to demonstrate exacerbation of cutaneous lesions characteristic of PCT. It was not until the patient started the Heliocare supplement that he noticed a significant reduction in the number of skin lesions and associated pain.

$P$. leucotomos has reported benefit in the prevention of idiopathic photodermatoses such as polymorphous light eruption (PLE) and has even been reported to induce remission in a patient with subacute cutaneous lupus erythematosus [4] [5]. The protective effects of P. leucotomos in these conditions are thought to occur via blocked production of UV-induced reactive oxygen species as well as the prevention of other UV-induced cellular damage [4]. Similarly, antioxidants such as ascorbic acid increase sun tolerability in erythropoietic protoporphyria and decrease porphyrin-induced cellular phototoxicity [6] [7]. In some patients, a deficiency of ascorbic acid is thought to be a contributing factor to the development of PCT [8]. The similar pathogenic role of oxygen free radicals in PMLE and PCT suggests that the benefit of treatment with P. leucotomos could be reproduced in patients with PCT. This conclusion is further supported by the established role of antioxidants in reducing porphyrin induced sun damage. To our knowledge, this is the first reported case of PCT treated with P. leucotomos. However, when taken in context of the mechanism by which P. leucotomos reduces photosensitivity and its proven efficacy in other photodermatoses, we believe this case demonstrates the opportunity for an expanded therapeutic role of P. leucotomos in PCT.

Treatment of PCT with P. leucotomos offers many advantages over standard therapy alone. As in this case, PCT has been known to occur in patients with hepatitis $\mathrm{C}$ who are also on hemodialysis. The two main treatments of PCT, phlebotomy and antimalarials, both pose challenges in the hemodialysis patient; anemia often precludes the use of high volume phlebotomy and hydroxychloroquine works by mobilizing porphyrins from the liver, which are poorly cleared by dialysis. P. leucotomos represents a possible adjunctive treatment for refractory PCT in patients on hemodialysis.

Additionally, the lack of adverse effects associated with $P$. leucotomos makes it an attractive therapeutic option in patients with multiple comorbid medical conditions. This favorable safety profile has been demonstrated in the medical literature. For example, in one study P. leucotomos was used to treat 57 cases of idiopathic photodermatoses with no observed side effects [9]. An additional benefit of $P$. leucotomos is that it is easily obtainable and available as a supplement from various commercial retailers without a prescription. The favorable side effect profile and availability of this agent may translate to increased patient compliance and satisfaction.

Although we describe an isolated case, the sustained improvement demonstrated by our patient along with the favorable qualities of Heliocare leads to our hopeful suggestion that $P$. leucotomos may be an effective adjunctive treatment in the setting of porphyria cutanea tarda. 


\section{References}

[1] Frank, J. and Poblete-Gutierrez, P.A. (2012) Porphyria. In: Bolognia, J.L., Jorizzo, J.L. and Schaffer, J.V., Eds., Dermatology, 3rd Edition, Elsevier Saunders, St. Louis, MO, 717-721.

[2] Gonzalez, S. and Patha, M.A. (1996) Inhibition of Ultraviolet-Induced Formation of Reactive Oxygen Species, Lipid Peroxidation, Erythema and Skin Photosensitization by Polypodium leucotomos. Photodermatology Photoimmunology \& Photomedicine, 12, 45-46. https://doi.org/10.1111/j.1600-0781.1996.tb00175.x

[3] Philips, N., Smith, J., Keller, T. and Gonzalez, S. (2003) Predominant Effects of Polypodium leucotomos on Membrane Integrity, Lipid Peroxidation, and Expression of Elastin and Matrixmetalloproteinase-1 in Ultraviolet Radiation Exposed Fibroblasts, and Keratinocytes. Journal of Dermatological Science, 32, 1-9. https://doi.org/10.1016/S0923-1811(03)00042-2

[4] Tanew, A., Radakovic, S., Gonzalez, S., Venturini, M., et al. (2012) Oral Administration of a Hydrophilic Extract of Polypodium leucotomos for the Prevention of Polymorphic Light Eruption. Journal of American Academy of Dermatology, 66, 58-62. https://doi.org/10.1016/j.jaad.2010.09.773

[5] Breithaupt, A.D. and Jacob, S.E. (2012) Subacute Cutaneous Lupus Erythematosus: A Case Report of Polypodium leucotomos as an Adjuvant Therapy. Cutis, 89, 183 184.

[6] Boffa, M.J., Ead, R.D., Reed, P. and Weinkove, C. (1996) A Double-Blind, PlaceboControlled, Crossover Trial of Oral Vitamin C in Erythropoietic Protoporphyria. Photodermatology Photoimmunology \& Photomedicine, 12, 27-30. https://doi.org/10.1111/j.1600-0781.1996.tb00240.x

[7] Böhm, F., Edge, R., Foley, S., Lange, L., et al. (2001) Antioxidant Inhibition of Porphyrin-Induced Cellular Phototoxicity. Journal of Photochemistry and Photobiology B: Biology, 65, 177-183. https://doi.org/10.1016/S1011-1344(01)00259-7

[8] Sinclair, P.R., Gorman, N., Shedlofsky, S.I., Honsinger, C.P., et al. (1997) Ascorbic Acid Deficiency in Porphyria Cutanea Tarda. Journal of Laboratory and Clinical Medicine, 130, 197-201. https://doi.org/10.1016/S0022-2143(97)90096-2

[9] Caccialanza, M., Recalcati, S. and Piccinno, R. (2011) Oral Polypodium Leucotomos Extract Photoprotective Activity in 57 Patients with Idiopathic Photodermatoses. Giornale Italiano di Dermatologia e Venereologia, 146, 85-87.

Submit or recommend next manuscript to OALib Journal and we will provide best service for you:

- Publication frequency: Monthly

- 9 subject areas of science, technology and medicine

- Fair and rigorous peer-review system

- Fast publication process

- Article promotion in various social networking sites (LinkedIn, Facebook, Twitter, etc.)

- Maximum dissemination of your research work

Submit Your Paper Online: Click Here to Submit

Or Contact service@oalib.com 